\title{
COMPARAÇÃO DOS NÍVEIS DE FLEXIBILIDADE ENTRE IDOSAS PRATICANTES DE GINÁSTICA LOCALIZADA E HIDROGINÁSTICA
}

\author{
COMPARISION OF FLEXIBILITY LEVELS AMONG ELDERLY WOMEN PRACTITIONERS \\ OF LOCATED GYMNASTICS AND HYDROGYMNASTICS
}

\author{
Aline Amorim Assunção ${ }^{a^{*}}$, João Carlos ${ }^{b^{*}}$, Riane de Paula Souza ${ }^{* *}$, Gabriel Andrade Paz $^{\mathrm{d}^{* *}}$, \\ Marianna de Freitas Maia ${ }^{\mathrm{e}^{* *}}$, Vicente Pinheiro Lima ${ }^{\mathrm{F}^{*}}$ \\ aassuncao_aline@hotmail.com, bdinhopaz@gmail.com, criane_paulas@hotmail.com, dgabriel.andrade.paz@gmail.com, \\ emariannamaia2@gmail.com, fprofessorvicentelima@gmail.com \\ *Universidade Castelo Branco - Rio de Janeiro (RJ), Brasil \\ **Universidade Federal do Rio de Janeiro - Rio de Janeiro (RJ), Brasil
}

Data de recebimento do artigo: 25/03/2015

Data de aceite do artigo: 17/09/2015

\section{RESUMO}

Introdução: $\mathrm{O}$ exercício físico é um importante fator de promoção da saúde, imprescindível para um envelhecimento saudável. Evidências sugerem que o envolvimento em programas de exercícios previne e/ ou minimiza os efeitos deletérios do envelhecimento. Objetivo: Comparar os ganhos de flexibilidade entre idosas praticantes de ginástica localizada (GGL) e hidroginástica (GH). Método: A amostra foi composta por 60 idosas, distribuídas aleatoriamente em três grupos: 20 idosas no GGL (65 $\pm 3,15$ anos); 20 idosas no GH (65 $\pm 3,1$ anos); e 20 idosas no grupo controle (GC) $(65 \pm 3,01$ anos). A avaliação da flexibilidade foi feita por meio da mensuração da amplitude articular nos movimentos abdução de ombros (AbdO) e flexão de quadril (FQ), utilizando o método da goniometria. Resultado: No movimento AbdO, o GGL obteve $168,16 \pm 4,3^{\circ}$, o GH $166,74 \pm 4,32^{\circ}$ e o grupo GNP obteve $136,84 \pm 17,79^{\circ}$, sendo GGL e GH significativamente maiores que GNP. Na FQ, o GGL obteve $79,26 \pm 3,72^{\circ}$, GH $80,21 \pm 4,59^{\circ}$ e GC $87,63 \pm 2,52^{\circ}$; da mesma forma, GGL e GH obtiveram diferença significativa em relação ao GC. Conclusão: A prática regular dos dois tipos de ginástica pode promover ganhos de amplitude de idosas nas articulações estudadas.

Palavras-chave: Flexibilidade; exercício; envelhecimento.

\section{ABSTRACT}

Introduction: Physical exercise is an important factor for health promotion, essential for healthy aging. Evidence suggests that engaging in exercise programs prevents and/or minimizes the deleterious effects of aging. Purpose: To compare the flexibility gain among older practitioners of located gymnastics (GLG) and hydrogymnastics $(\mathrm{GH})$. Method: The sample consisted of 60 elderly women, randomly assigned to three groups: 20 elderly in GLG (65 \pm 3.15 years); 20 elderly in GH (65 \pm 3.1 years); and 20 elderly in the control group (CG) $(65 \pm 3.01$ years). The flexibility assessment was made by measuring the range of motion in shoulder abduction (AbdS) and hip flexion (HF) movements through the goniometry method. Result: During AbdS, GLG showed $168.16 \pm 4.3^{\circ}$, GH $166.74 \pm 4.32^{\circ}$, and the GNP group showed $136.84 \pm 17.79^{\circ}$, demonstrating that GLG and GH data was significantly greater than GNP. In HF, the GLG obtained $79.26 \pm 3.72^{\circ}$, GH $80.21 \pm 4.59^{\circ}$, and CG $87.63 \pm 2.52^{\circ}$; GLG and GH also showed significant difference compared to the CG. Conclusion: The regular practice of both types of exercise can promote amplitude gains in the studied joints of elderly women.

Keywords: Flexibility; exercise; aging. 


\section{Introdução}

O número de idosos tem aumentado em termos relativos e absolutos em quase todos os países do mundo. No Brasil, segundo dados do Instituto Brasileiro de Geografia e Estatística (IBGE), o grupo de idosos de 60 anos ou mais será maior que o grupo de crianças até 14 anos em $2030^{1}$. Com o envelhecimento o indivíduo sofre diversas alteraçóes morfológicas e fisiológicas, tais como atrofia muscular, declínio funcional, descalcificação óssea, aumento da espessura da parede de vasos, aumento do nível de gordura corporal total e diminuição da capacidade coordenativa ${ }^{2}$.

Essas modificaçóes acabam gerando declínio dos níveis de atividade física e de vida diária ${ }^{3}$, além de redução da flexibilidade decorrente da falta de mobilidade das articulaçóes, associada ao envelhecimento do tecido conjuntivo, tendóes, ligamentos e cápsulas articulares ${ }^{4}$. Essa diminuição da flexibilidade pode tornar o idoso dependente de outras pessoas ou de algum tipo de assistência na realização das atividades da vida diária ${ }^{5}$. Ainda nesse sentido, diferentes autores parecem concordar que as implicaçóes das perdas de força e flexibilidade aliadas ao envelhecimento fisiológico normal apresenta uma tendência à inatividade física, o que compromete as principais capacidades físicas do idoso ${ }^{6-8}$.

O exercício físico é um importante fator de promoção da saúde, imprescindível para um envelhecimento saudável ${ }^{3}$. Evidências sugerem que o envolvimento em programas de exercícios previne e/ou minimiza os efeitos deletérios do envelhecimento, uma vez que um programa de atividade física adequado pode retardar a progressão do processo degenerativo que acompanha o envelhecimento ${ }^{10}$. Entre as diferentes atividades recomendadas para o idoso temos a hidroginástica, constituída por exercícios específicos baseados no aproveitamento da água como sobrecarga, com intensidade moderada e formato aeróbico, porém, sem enfatizar o trabalho de força e flexibilidade; e a ginástica localizada, que é uma modalidade de treinamento resistido que ocorre com aulas coreografadas ${ }^{11,12}$. Apesar das diferenças metodológicas entre as duas atividades, ambas parecem promover aspectos da aptidão física importantes para a saúde de idosos ${ }^{2,13}$.

Por outro lado, evidências na literatura sobre o desenvolvimento e manutenção da flexibilidade de idosos nas aulas de hidroginástica e ginástica localizada são escassos. Dessa forma, torna-se interessante identificar qual modalidade de exercício físico associa-se com maiores níveis de flexibilidade em articulaçóes como o ombro e joelho, que são primordiais para a execução de grande parte dos movimentos realizados durante a realização das atividades de vida diária ${ }^{14}$.
Logo, este estudo teve como objetivo avaliar a flexibilidade de mulheres idosas praticantes de hidroginástica e ginástica localizada com a flexibilidade de idosas não praticantes.

\section{Métodos}

\section{Amostra}

A amostra foi composta por 60 mulheres idosas, voluntárias, aparentemente saudáveis, não praticantes de atividade física sistematizada há no mínimo seis meses, oriundas da Zona Oeste da cidade do Rio de Janeiro. As participantes foram subdivididas em três grupos: praticantes de hidroginástica $(\mathrm{GH})$ (20 idosas com idade entre $65 \pm 3,15$ anos), praticantes de ginástica localizada (GGL) (20 idosas com idade 65 $\pm 3,10$ anos) e o grupo controle (GC), que não praticou atividade física (20 idosas com $65 \pm 3,01$ anos). Foi adotado como critério de inclusão para os grupos GC, GH e GGL não praticar atividades físicas regulares há no mínimo um ano. Foram adotados como critérios de exclusão para GH e GGL: apresentar problemas osteomioarticulares ou metabólicos que limitassem ou contraindicassem a prática dos exercícios programados; quadro de infarto há pelo menos dois anos; insuficiência cardíaca, cardiopatia isquêmica ou angina instável; participação em outros programas regulares de exercícios; uso de substâncias ergogênicas.

Para atender a lei 466/12 do Conselho Nacional de Saúde, as participantes assinaram o termo de participaçáo livre e esclarecido pela diretoria do projeto Vila Olímpica Ary de Carvalho e participantes do estudo direção. Este estudo foi devidamente aprovado pelo Comitê de Ética em Pesquisa da Universidade Castelo Branco sob o número 006/2010.

\section{Procedimentos}

Neste estudo foi realizada uma pesquisa descritiva com característica observacional e delineamento longitudinal ${ }^{15}$. A avaliação da flexibilidade foi feita antes e após um período de 20 semanas de treinamento. Em um dia previamente combinado as idosas foram avaliadas antes de iniciar as aulas de ginástica localizada e hidroginástica, e após um período de 20 semanas de treinamento as participantes foram retestadas.

\section{Protocolo de treinamento}

O programa de exercícios nas aulas de ginástica localizada foi composto por exercícios 
multiarticulares para o membro superior e inferior, realizando em média 8-10 exercícios conjugados, com intervalo de recuperação ativo entre as séries, realizando entre 12 a 20 repetiçóes com cargas submáximas. Um programa similar foi adotado nas aulas de hidroginástica. As idosas realizaram duas aulas por semana com duração média de 50-60 minutos.

\section{Avaliação da flexibilidade}

A mensuração da amplitude articular foi realizada com o goniômetro Carci (ind. E com. De apar. Cirúr. e ortop. Ltda), com a técnica em que os resultados são expressos em ângulos formados entre dois segmentos que se opõem na articulação. Foram utilizados os testes angulares de goniométrica do protocolo Labifie ${ }^{16}$. A abduçáo de ombro (AbdO) foi medida solicitando ao voluntário para ficar de pé com o braço abduzido; localizado o acrômio e medindo aproximadamente dois dedos abaixo dele, segue em direção à espinha da escápula o teste da flexão do quadril (FQ), que foi realizado com o voluntário em decúbito dorsal, sendo solicitado a flexão do quadril com o joelho em extensão na maior amplitude do movimento possível.

\section{Tratamento estatístico}

A estatística descritiva foi realizada pela média e desvio padrão dos resultados. O teste de ShapiroWilk foi aplicado para verificar a normalidade dos dados. Em seguida, a One way Anova para medidas repetidas seguidas pelo post hoc de Tukey, quando necessário, foi aplicado. Foi adotado o nível de significância de $\mathrm{p}<0,05$ para todos os procedimentos estatísticos. A análise estatística foi realizada no software SPSS 20.0.

\section{Resultados}

De acordo com os resultados, houve diferença significativa no movimento de abdução de ombros, que foi maior no grupo GGL ( $\mathrm{p}=0,001)$ sobre os grupos $\mathrm{GH}$ $(\mathrm{p}=0,02)$ e GC. Adicionalmente, foi observada diferença entre o grupo GH e GC.

Também foi observada diferença significativa no movimento de flexão de quadril, que foi maior no grupo GC sobre os grupos GGL $(\mathrm{p}=0,002)$ e $\mathrm{GH}$ $(\mathrm{p}=0,001)$, assim como verificou-se diferença entre o grupo GH sobre o grupo GGL.
Tabela 1: Média e desvio-padrão (DP) dos valores da goniometria no movimento de abdução do ombro e flexão do quadril.

\begin{tabular}{lcc} 
& $\begin{array}{c}\text { Abduçáo de ombros } \\
\text { (graus) }\end{array}$ & $\begin{array}{c}\text { Flexão do quadril } \\
\text { (graus) }\end{array}$ \\
\hline Hidroginástica & $166,7(4,3)^{*}$ & $80,2(4,5)^{*} ¥$ \\
\hline Ginástica & $168,1(3,3)^{*}$ & $79,2(3,7)^{*}$ \\
\hline Não praticante & $136,8(17,9)$ & $87,6(2,5)$ \\
\hline
\end{tabular}

* Diferença significativa em relaçấo ao grupo de nâo praticantes de atividades físicas.

$¥$ Diferença significativa para o grupo de praticantes de ginástica.

\section{Análise e discussão dos resultados}

O principal achado do nosso estudo foi que a modalidade de ginástica (GGL) promove maiores ganhos de amplitude articular da cintura escapular no movimento de abdução de ombros em relaçẫo à modalidade hidroginástica $(\mathrm{GH})$ e aos que não praticaram nenhuma atividade (GC). Em relação ao movimento de flexão de quadril, o grupo de náo praticantes apresentou maiores ganhos de amplitude articular sobre as modalidades de ginástica e hidroginástica.

Os resultados encontrados nos indivíduos do grupo GGL corroboram com um estudo que analisou os benefícios da modalidade sobre a postura e a flexibilidade de idosas ${ }^{17}$. Nesse estudo, foi avaliado o movimento de flexão de tronco por meio do teste de sentar e alcançar. Foi observado que com a intervenção da ginástica localizada aumentou a flexibilidade e a mobilidade da articulação em questáo. Apesar de articulaçóes diferentes, ambos promoveram ganhos de flexibilidade e mobilidade articular. Outros autores estudaram a influência da atividade física sobre a flexibilidade em idosas e chegaram à conjunta conclusão que a intervençáo de atividade física traz ganhos em curto e longo prazo ${ }^{18-20}$. Não existem estudos anteriores que analisaram a flexibilidade e mobilidade da cintura escapular e cintura pélvica nos movimentos de abdução de ombros e flexão de quadril por meio da técnica de goniometria.

Os resultados encontrados nos indivíduos do grupo GH corroboram com estudos anteriores, que demonstraram que exercícios de hidroginástica promovem ganhos de mobilidade articular da cintura pélvica e cintura escapular. Nesse estudo, foram selecionadas 74 mulheres idosas que nunca praticaram exercícios físicos, e foram divididas em dois grupos com o mesmo número de indivíduos. No grupo um (G1) foram ministradas aulas de hidroginástica com volume semanal de duas vezes e volume de treinamento de 45 minutos por 12 semanas. 
O grupo dois (G2) foi o controle, os indivíduos não praticaram exercícios físicos. Foram realizados os testes de alcançar atrás das costas para mensuração de mobilidade articular da cintura escapular e sentar e alcançar mobilidade de tronco e cintura pélvica. Após 12 semanas, foi observado aumento de mobilidade da cintura escapular em ambos os grupos - sendo superior no G1 -, e aumento de mobilidade de tronco e cintura pélvica em ambos os grupos, sendo inferior no $\mathrm{G}^{21}$. Em outro estudo foram selecionadas 36 mulheres idosas que nunca praticaram atividades físicas e foram submetidas ao treinamento de hidroginástica com volume semanal de três vezes em dias alternados com volume de treinamento de 50 minutos por 12 semanas. Foi observada melhora na flexibilidade e mobilidade da cintura pélvica em relação aos movimentos de flexão e extensão do quadril e em relação ao início e fim do programa de intervenção, medidos por meio de fita métrica e goniômetro ${ }^{13}$. Não parece verdade que a intervenção de hidroginástica melhora a flexibilidade e mobilidade da cintura escapular em relaçăo ao movimento de abdução de ombros em praticantes idosos de ginástica e de indivíduos nâo praticantes de exercício físico. Outro estudo observou diferente variável, que idosos praticantes de hidroginástica possuem maior força isométrica de preensão manual do lado esquerdo sobre os praticantes de ginástica ${ }^{22}$.

Diferente estudo analisou durante oito semanas 19 idosos, que praticaram um treinamento de força três vezes por semana, com sete exercícios totais e com método alternado por segmento. Observou-se manutenção e aumento na flexibilidade e mobilidade articular do ombro (flexão e extensão), do cotovelo (flexão e extensão), do quadril (flexão e extensão) e do joelho (flexão) ${ }^{23}$. Outro estudo analisou durante 58 semanas 32 idosos, que realizaram 174 sessóes de treinamento de força três vezes por semana, com duração entre 50 e 55 minutos total; não foi observado aumento significativo na flexibilidade dos indivíduos testados ${ }^{24}$.

A reserva funcional de certos indivíduos de idade avançada é bem reduzida, e as perdas de força, flexibilidade e agilidade podem representar a diferença entre uma vida autônoma ou não ${ }^{25}$. Ao decorrer do envelhecimento vamos perdendo importantes funçóes como força, flexibilidade e mobilidade, além da perda de massa livre de gordura (óssea e muscular) e ganho de massa de gordura.

Fator de grande importância relacionado ao envelhecimento é que naturalmente ocorre perda de agilidade e da massa muscular e óssea, e elevado número de quedas e fraturas nessa fase ${ }^{25}$. Diferente estudo observou que em mulheres de meia idade (pós-menopausa) o aumento da densidade mineral óssea foi maior em praticantes de hidroginástica ${ }^{26}$. Em relação à massa corporal (proporção de gordura e peso corporal total; proporçáo de gordura nos braços; proporçáo de gordura nas pernas; proporção de gordura no tronco e massa magra), um estudo anterior demonstrou que tanto a ginástica aeróbica quanto a hidroginástica apresentaram melhoras, e não foram observadas diferenças significativas entre as duas modalidades ${ }^{27}$. $\mathrm{O}$ número de quedas foi consideravelmente menor em indivíduos idosos ativos ${ }^{28}$.

Diversas variáveis atuam diretamente para a perda de amplitude articular saudável, e duas delas são encontradas mais facilmente, a falta de mobilidade ou elasticidade $\operatorname{articular}^{30}$ e o encurtamento muscular ${ }^{19}$.

A literatura apresenta poucos estudos desse gênero com idosos. Analisando a especificidade de cada modalidade e dos movimentos articulares avaliados e comparando-as entre si e com indivíduos sedentários, podemos elucidar algumas possíveis questões. Exercícios em meio líquido desenvolvem maiores ganhos de amplitude articular da regiáo tóraco-lombar aos pés comparado aos membros superiores ${ }^{13}$. Uma possível resposta é que devido a sua posição ao meio em que é gerada a resistência e maior amplitude de alongamento ao movimento realizado, a água fica próxima à região meso-esternal. Exercícios em meio sólido possibilitam maior liberdade aos movimentos de membros superiores, aproveitando as amplitudes máximas de cada movimento; em contrapartida, limita determinados movimentos com hiperflexão e hiperextensão do quadril.

Maiores estudos acerca dessa temática e desse perfil etário se faz necessário para um melhor entendimento sobre os mecanismos mecano-fisio-articulares envolvidos. Uma adequada variação das modalidades e manipulação das variáveis metodológicas promove melhor autonomia na população idosa. Entretanto, há necessidade de um número maior de estudos que avaliem os efeitos aqui abordados e outros da hidroginástica sobre a aptidáo física dos idosos.

\section{Limitações do estudo}

Foram comparados exercícios em dois ambientes diferentes (solo e água), e nos exercícios de hidroginástica utilizou-se aumento de cargas por meio de escala de percepção subjetiva de esforço. O sexo e a faixa etária são fatores limitantes para trabalhar com intensidades altas nas atividades propostas, em que como mecanismo de defesa os indivíduos utilizam cargas submáximas.

\section{Conclusão}

Programas de ginástica localizada e hidroginástica corroboram para a melhoria e manutenção da aptidão 
física em idosos. Podemos concluir que ambas melhoram movimentos importantes para a vida diária dos indivíduos e que ao passar dos anos vão perdendo sua melhor funçáo. A ginástica localizada promove maior ganho de amplitude no movimento de abduçáo do ombro e consequente maior mobilidade da cintura escapular; já a hidroginástica promove maior ganho de amplitude no movimento de flexão de quadril e consequentemente maior mobilidade da cintura pélvica.

\section{Referências}

1. Instituto Brasileiro de Geografia e Estatística [base de dados na Internet]. [acesso em 2014 Nov 10]. Disponível em: http://www.ibge.gov.br

2. Liebl EC, Mascarenhas LPG, Gonçalves R, Lima VA, Souza WB, Grzelczak MT, et al. Comparação dos efeitos da ginástica localizada e musculação nos níveis de resistência muscular localizada e força em mulheres. Revista Acta Brasileira do Movimento Humano. 2014;4(1):1-12.

3. American College of Sports Medicine. ACSM Position Stand: exercise and physical activity for older adults. Med Sci Sports Exer. 2009;41(1):1510-1530.

4. Weineck J. Biologia do esporte. $7^{\mathrm{a}}$ ed. São Paulo: Manole; 2005.

5. Andreotti RA, Okuma SS. Validação de uma bateria de testes de atividades da vida diária para idosos fisicamente independentes. Rev paul educ fís. 1999;13(1):36-66.

6. Hunter SK, Thompson MW, Adams RD. Reaction time, strength, and physical activity in women aged 20-89 years. J Aging and Phys Act. 2001;9(1):32-42.

7. Matsudo SMM. Envelhecimento e atividade física. $1^{\mathrm{a}} \mathrm{ed}$. Londrina: Midiograf; 2001.

8. Holland GJ, Tanaka K, Shigematsu R, Nakagaichi M. Flexibility and physical functions of older adults: a review. J Aging and Phys Act. 2002;10(1):169-2006.

9. Katula JA, Rejeski WJ, Anthony PM. Enhancing quality of life in older adults: a comparison of muscular strength and power training. Health Qual Life Outcomes. 2008;6:1-8.

10. Hurley SK, Hagberg J. Optimizing health in older person: aerobic or strength? Exerc Sport Sci Rev. 1998;26(1):61-89.

11. Kruel LFM. Alterações fisiológicas e biomecânicas em indivíduos praticando exercícios de hidroginástica dentro e fora da água [tese de doutorado em Educaçáo Física]. Santa Maria: Universidade Federal de Santa Maria. Centro de Educação e Desportes, 2000.

12. Oliveira CEP, Moreira OC, Pereira LS, Doimo LA. Efeito de oito semanas de treinamento de ginástica localizada sobre a composição corporal de mulheres sedentárias. Rev bras Cineamtropom e Mov. 2013;21(3):135-141.

13. Passos BMA, Souza LHR, Silva FM, Lima RR, Oliveira RJ. Contribuiçôes da hidroginástica nas atividades da vida diária e na flexibilidade de mulheres idosas. Rev educ fis. 2008;19(1):71-76.
14. Paz GA, Maia MF, Lima VP, Miranda H. Efeito do método agonista-antagonista versus tradicional sobre o volume e ativação muscular. Rev bras ativ fís saúde. 2014;19:54-63.

15. Thomas JR, Nelson JK, Silverman SJ. Métodos de pesquisa em atividade física. $6^{\mathrm{a}}$ ed. Porto Alegre: Artmed; 2012.

16. Dantas EHM. Alongamento e flexionamento. $5^{\mathrm{a}} \mathrm{ed}$. Rio de Janeiro: Shape; 2005.

17. Lima HCO, Aguiar JB, Paredes PFM, Gurgel LA. Avaliação dos benefícios da ginástica localizada sobre a postura e a flexibilidade de mulheres na terceira idade. Rev Bras Educ Fís Esporte. 2010;24(4):525-34.

18. Canderolo JM, Caromano FA. Efeito de um programa de hidroterapia na flexibilidade e na força muscular de idosas. Rev bras fisioter. 2007;11(4):303-309.

19. Cristopoliski F, Sarraf TA, Dezan VH, Provensi CLG, Rodacki ALF. Efeito transiente de exercícios de flexibilidade na articulação do quadril sobre a marcha de idosas. Rev Bras Med Esporte. 2008;14(2):139-144.

20. Geraldes AAR, Albuquerque RB, Soares RM, Carvalho J, Farinatti PVT. Correlação entre flexibilidade das articulaçóes glenoumerais e coxofemorais e o desempenho funcional de idosas fisicamente ativas. Rev bras fisioter. 2008;12(4):274-282.

21. Alves RV, Mota J, Costa MC, Alves JGB. Aptidão física relacionada à saúde de idosos: influência da hidroginástica. Rev Bras Med Esporte. 2004;10(1):31-37.

22. Kura RV, Ribeiro LSP, Niquetti R, Tourinho Filho H. Nível de atividade física, IMC e índices de força muscular estática entre idosas praticantes de hidroginástica e ginástica. Revista Brasileira de Ciências do Envelhecimento Humano. 2004;1(2):30-40.

23. Gonçalves R, Gurjāo ARD, Gobbi S. Efeitos de oito semanas do treinamento de força na flexibilidade de idosos. Rev bras cineantropom desempenho hum. 2007;9(2):145-153.

24. Rebelatto JR, Calvo JJ, Orejuela JR, Portillo JC. Influência de um programa de atividade física de longa duração sobre a força muscular manual e a flexibilidade corporal de mulheres idosas. Rev bras fisioter. 2006;10(1):127-132.

25. Monteiro WD, Amorim PRS, Farjalla R, Farinatti PVT. Força muscular e características morfológicas de mulheres idosas praticantes de um programa de atividades físicas. Rev bras ativ fís saúde. 1999;4(1):20-28.

26. Silva VF, Matsuura C. Prevenção de quedas em idosos: efeitos da prática regular de atividades físicas sobre o estado cognitivo e a prevenção de quedas em idosos. Fitness \& Performance Journal. 2002;1(3):39-45.

27. Balsamo S, Simão R, Marques MB, Paula AP, Borges JL. Comparação da densidade mineral óssea em mulheres praticantes de hidroginática e sedentárias na pós-menopausa. Fitness \& Performance Journal. 2006;5(4):210-214.

28. Melo GF, Giavoni A. Comparação dos efeitos da ginástica aeróbica e da hidroginástica na composição corporal de 
mulheres idosas. Revista Brasileira de Ciência e Movimento. 2004;12(2):13-18.

29. Liu-Amborse T, Khan KM, Eng JJ, Jansesn PA, Lord SR, Mickav $H$. Resistance and agility training reduce fall risk in women aged 75 to 85 with low bone mass: a 6-month randomized, controlled trial. Journal of the American Geriatric Society. 2004;52(1):657-665.

30. Dantas EHM, Pereira SAM, Aragão JC, Ota AHA. A preponderância da diminuição da mobilidade articular ou da elasticidade muscular na perda da flexibilidade no envelhecimento. Fitness \& Performance Journal. 2002;1(3):12-20.

\section{Como citar este artigo:}

Assunção AA, Carlos J, Souza RP, Paz GA, Maia MF, Lima VP. Comparação dos níveis de flexibilidade entre idosas praticantes de ginástica localizada e hidroginástica. Rev. Aten. Saúde. 2016;14(47): 19-24. 